02

\title{
Влияние молекулярного веса поливинилпирролидона на структуру, спектральные и нелинейно-оптические свойства композиционных материалов, содержащих наночастицы CdS/ZnS
}

\author{
(C) С.К. Евстропьев ${ }^{1}$, А.С. Кулагина ${ }^{1,2}$, К.С. Евстропьев ${ }^{1}$, \\ Е.В. Колобкова ${ }^{1}$, Н.В. Никоноров ${ }^{1}$, И.П. Сошников ${ }^{2,3}$, \\ К.В. Орешкина ${ }^{1}$, А.И. Хребтов ${ }^{2}$ \\ ${ }^{1}$ Университет ИТМО, \\ 197101 Санкт-Петербург, Россия \\ ${ }^{2}$ Санкт-Петербургский национальный исследовательский Академический университет РАН, \\ 194021 Санкт-Петербург, Россия \\ ${ }^{3}$ Физико-технический институт им. А.Ф.Иоффре, 194064 Санкт-Петербург, Россия \\ e-mail: evstropiev@bk.ru
}

Поступила в редакцию 21.06.2018 г.

В окончательной редакции 27.07.2018 г.

\begin{abstract}
Представлены экспериментальные результаты исследований влияния молекулярного веса поливинилпирролидона на структуру, спектральные и нелинейно-оптические свойства композиционных материалов (золей и покрытий), содержащих наночастицы $\mathrm{CdS} / \mathrm{ZnS}$. Наночастицы $\mathrm{CdS} / \mathrm{ZnS}$ были получены жидкостным коллоидно-химическим методом в присутствии поливинилпирролидона. Установлено, что размер молекул поливинилпирролидона оказывает сильное влияние на размер формирующихся халькогенидных частиц, структуру материалов и их спектральные свойства.
\end{abstract}

DOI: $10.21883 /$ OS.2018.11.46818.174-18

\section{Введение}

Квантовые точки (КT) $\mathrm{CdS}$ перспективны для многих современных приложений - солнечной энергетики, фотоники и оптоэлектроники, нелинейной оптики, медицины [1-8]. Поэтому разработка методов синтеза и стабилизации этих КТ является актуальной задачей.

Жидкостные методы синтеза КТ технологичны и широко используются [8-13]. В этих методах для стабилизации формирующихся в растворах наночастиц используются некоторые органические вещества или органические полимеры. Тип органического стабилизатора и его концентрация являются важными факторами, оказывающими сильное влияние на стабильность и свойства КТ.

Поливинилпирролидон (ПВП) является водорастворимым полимером, широко используемым для стабилизации различных наночастиц [7-22]. Молекулы ПВП обеспечивают значительное усиление люминесцентных свойств нанокристаллов $\mathrm{CdS}$ [16] и препятствуют их росту и агрегации [15]. Экспериментальные результаты показали, что стабилизирующий эффект и высокие люминесцентные свойства КТ $\mathrm{ZnS}$ [13] и $\mathrm{PbS}$ [9-11,23], стабилизированных ПВП, особенно ярко проявляются при определенных оптимальных концентрациях этого полимера.

Молекулярный вес растворимого полимера является одним из важных факторов, влияющих на стабильность
КТ и их оптические свойства. Размер молекул полимера может влиять на толщину оболочки, окружающей наночастицы и вязкость коллоидного раствора. Поэтому молекулярный вес полимерного стабилизатора является важным параметром при оптимизации тех или иных характеристик КТ, и исследование его влияния на структуру и свойства наночастиц является актуальной задачей.

В $[14,18]$ было показано, что нанокристаллы CdS обладают низкой фотостойкостью и устойчивостью к окислению. Для увеличения стабильности КТ CdS и улучшения их люминесцентных характеристик на их поверхность наносят тонкие покрытия из сульфида цинка $[10,19-21]$.

В настоящей работе описаны результаты экспериментальных исследований по влиянию молекулярного веса ПВП на структуру, спектральные и нелинейнооптические свойства $\mathrm{CdS} / \mathrm{ZnS}$ золей и $\mathrm{CdS} / \mathrm{ZnS}-П В П$ композиционных покрытий.

\section{Синтез материалов и методика экспериментов}

В работе в качестве исходных материалов для получения $\mathrm{CdS} / \mathrm{ZnS}$ золей были использованы водные растворы $\mathrm{Cd}\left(\mathrm{NO}_{3}\right)_{2} \quad\left(6.5 \cdot 10^{-2} \mathrm{M}\right), \quad \mathrm{Zn}\left(\mathrm{NO}_{3}\right)_{2}$ $\left(1,3 \cdot 10^{-1} \mathrm{M}\right)$ и $\mathrm{Na}_{2} \mathrm{~S}\left(4.2 \cdot 10^{-2} \mathrm{M}\right)$. Для стабилизации формирующихся в растворе КТ использовался 
ПВП различного молекулярного веса: высокомолекулярный $\left(M_{s}=1300000\right.$; Sigma-Aldrich $)$ и низкомолекулярный $\left(M_{s}=10000 ; \mathrm{K} 15\right.$; BioChemica $)$. Порошки ПВП растворялись в пропаноле-2 при комнатной температуре.

На первой стадии синтеза раствор $\mathrm{Cd}\left(\mathrm{NO}_{3}\right)_{2}$ смешивался при комнатной температуре с раствором ПВП в пропаноле-2. Затем к полученной смеси небольшими порциями при интенсивном перемешивании добавлялся водный раствор $\mathrm{Na}_{2} \mathrm{~S}$. Полученный раствор перемешивался в течение $10 \mathrm{~min}$ с помощью магнитной мешалки. В результате химической реакции между сульфидом натрия и нитратом кадмия в присутствии ПВП происходило образование золя $\mathrm{CdS}$. Добавление раствора нитрата цинка к полученному золю ведет к взаимодействию между ионами $\mathrm{Zn}^{2+}$ и поверхностью частиц $\mathrm{CdS}$ и формированию на этой поверхности тонкого слоя $\mathrm{ZnS}$. Детали синтеза были описаны ранее в [10].

В результате синтеза были получены два золя, стабилизированные высокомолекулярным ПВП (золь 1) и низкомолекулярным ПВП (золь 2). Содержание ПВП в обоих золях составляло 2.1 масс.\%. Концентрация $\mathrm{CdS}$ в этих золях была одинакова и составляла $3.3 \cdot 10^{-3} \mathrm{M}$. Расчет содержания $\mathrm{CdS}$ основывался на данных о концентрациях ионов $\mathrm{Cd}^{2+}$ и $\mathrm{S}^{2-}$ в приготовленных растворах и известных литературных данных о растворимости CdS $\left(\Pi \mathrm{P}=1 \cdot 10^{-27}\right)$.

Полученные золи были использованы для формирования покрытий на поверхности шелочно-силикатного стекла (Menzel-Glazer, № 02 1102) методом погружения стеклянных образцов в раствор с последующим извлечением и сушкой при $50^{\circ} \mathrm{C}$ в течение $24 \mathrm{~h}$.

Спектры поглощения золей и стеклянных образцов с покрытиями измерялись на спектрофотометре Shimadzu UV-3600.

Измерение нелинейно-оптических характеристик выполнялось с помощью метода $z$-сканирования [24]. В оптической схеме использовалось излучение второй гармоники YAG: Nd-лазера в режиме моноимпульса (параметры импульса $\left.\lambda_{\text {ex }}=532 \mathrm{~nm}, \tau_{\text {pulse }}=5 \mathrm{~ns}, E_{\text {pulse }}=50 \mu \mathrm{J}\right)$, пучок имел гауссов профиль и фокусировался на образец линзой $(F=+15 \mathrm{~cm})$. Кварцевая кювета с $\mathrm{CdS} / \mathrm{ZnS}-$ ПВП золем перемещалась вдоль главной оптической оси с шагом $1 \mathrm{~mm}$, проходя последовательно положения до фокусной плоскости (отрицательные значения $z$ ) и после (положительные значения $z$ ). Входная и выходная энергии измерялись калиброванными фотометрами Ophir. Энергия на выходе из кюветы измерялась фотометром (OPHIR, PE9) в двух режимах „с закрытой апертурой“ и „с открытой апертурой“, что позволило отделить вклад нелинейной рефракции от нелинейного поглощения и рассеяния. Для оценки вклада нелинейного рассеяния использовались показания третьего приёмника (OPHIR, PD10), расположенного под углом к передней стенке кюветы с образцом (с возможностью варьирования угла в диапазоне от 10 до $85^{\circ}$ ). Толщина образца была выбрана с учетом критерия Релея

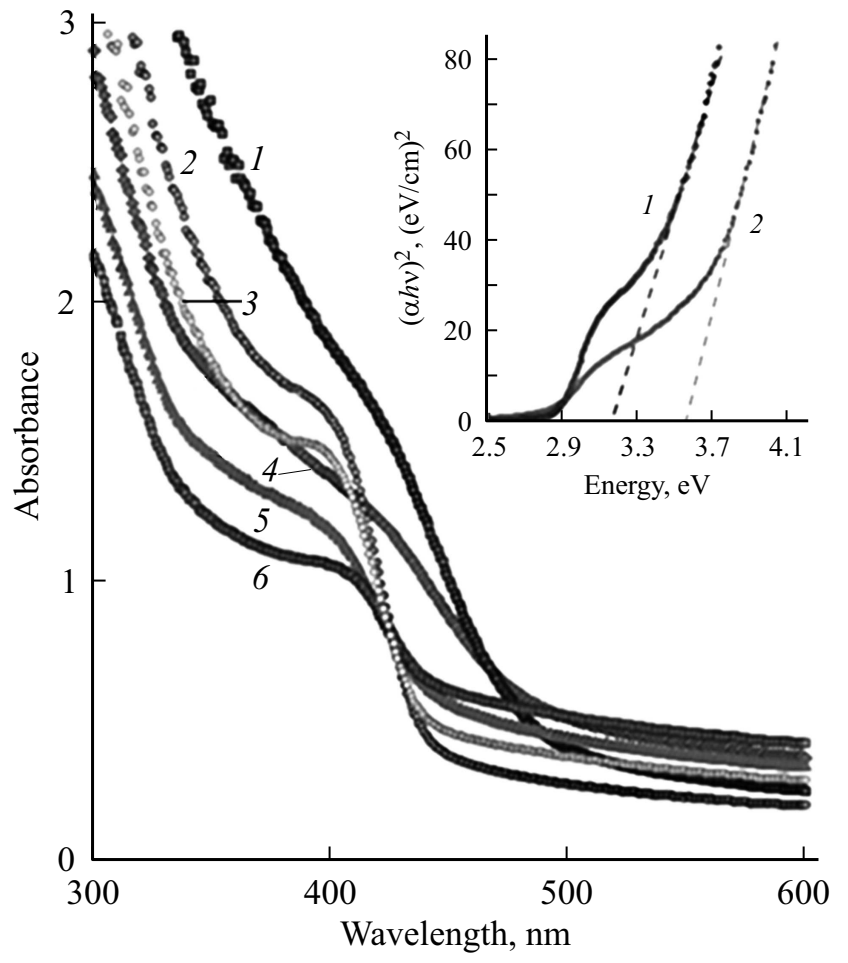

Рис. 1. Спектры поглощения свежеприготовленных золей 1 (кривая 1) и 2 (кривая 4) и этих золей после временной выдержки в течение $24 \mathrm{~h}$ (кривые 2 и 5 соответственно), в течение $48 \mathrm{~h}$ (кривые 3 и 6 соответственно). Врезка: Зависимости $(\alpha h v)^{2}=f(h v)$ для золей $\mathbf{1}$ и $\mathbf{2}$ после их временной выдержки в течение $24 \mathrm{~h}$.

и составляла $2 \mathrm{~mm}$. Режимы с открытой и закрытой апертурами позволили отделить вклад нелинейного поглощения и рассеяния от вклада нелинейной рефракции.

Морфология покрытий были исследована методом растровой электронной микроскопии (SEM) на приборе Carl Zeiss SUPRA 25.

\section{Результаты и обсуждение}

На рис. 1 представлены спектры поглощения свежеприготовленных золей 1 (кривая 1) и 2 (кривая 4) и этих золей после временных выдержек при комнатной температуре в течение $24 \mathrm{~h}$ (кривые 2 и 5 ) и $48 \mathrm{~h}$ (кривые 3 и 6). Оба золя содержали одинаковое количество $\mathrm{CdS}$ $\left(3.3 \cdot 10^{-3} \mathrm{M}\right)$, поэтому наблюдаемые различия в спектрах связаны с разницей в размерах сформировавшихся частиц и структурой коллоидных растворов. Форма приведенных спектров часто наблюдается в коллоидных материалах, содержащих CdS-частицы $([10,21])$. Видно, что золи демонстрируют высокое поглощение в УФ области спектра и характерные перегибы на спектрах в области $400-410 \mathrm{~nm}$, связанные с экситонным поглощением полупроводниковых нанокристаллов. 
Поглощение света в УФ диапазоне уменьшалось со временем при хранении золей при комнатной температуре. Наблюдаемые изменения в спектрах поглощения объясняются процессами эволюции наночастиц.

Макроскопические кристаллы $\mathrm{CdS}$ и $\mathrm{ZnS}$ являются прямозонными полупроводниками со значениями ширины запрещенной зоны 2.4-2.5eV [25] и $3.6 \mathrm{eV}$ [26] соответственно. Эти значения соответствуют длинам волн света $517 \mathrm{~nm}(\mathrm{CdS})$ и $345 \mathrm{~nm}(\mathrm{ZnS})$. Учитывая эти данные, нужно отметить относительно высокую прозрачность полученных золей в УФ области спектра (рис. 1). Наблюдаемое явление связано с малым размером образовавшихся кристаллов и соответственным изменением в ширине запрещенной зоны материалов.

Для расчета величин запрещенных зон полученных полупроводниковых нанокристаллов использовалось уравнение Таука [27], которое для прямозонных полупроводниковых может быть записано в следующем виде:

$$
(\alpha h v)^{2}=A\left(h v-E_{g}\right),
$$

где $h v-$ энергия фотона, $E_{g}$ - ширина запрещенной зоны полупроводниковой частицы, $A-$ постоянная, и $\alpha$ - коэффициент поглощения. Графики в координатах $(\alpha h v)^{2}=f(h v)$ позволяют определить $E_{g}$ путем линейной экстраполяции полученных зависимостей на ось $x$.

Зависимости $(\alpha h v)^{2}=f(h v)$ для золей 1 и 2 после их выдержки при комнатной температуре в течение $24 \mathrm{~h}$ показаны на вставке рис. 1 . Полученные значения $E_{g}$ для $\mathrm{CdS}$ нанокристаллов для золей 1 и 2 составили 3.1 и $3.6 \mathrm{eV}$ соответственно. Эти значения близки к данным, полученным в [20] для золей $\mathrm{CdS}$, синтезированных аналогичным методом и значительно выше благодаря квантово-размерному эффекту, чем значение $E_{g}$ макроскопических кристаллов $\mathrm{CdS}$.

В [28] были рассмотрены несколько теоретических моделей, описывающих увеличение ширины запрещенной зоны с уменышением размеров кристаллов $\mathrm{CdS}$. На основании модели, описанной в [29], для оценки диаметра $D$ наночастиц $\mathrm{CdS}$ в [28] была предложена следующая формула:

$$
D=3.062 / \sqrt{\Delta E_{g}},
$$

где $\Delta E_{g}=E_{g}^{\text {nanoparticle }}-E_{g}^{\text {bulk }}$. Диаметр наночастицы $D$ и значение $\Delta E_{g}$ выражены в этой формуле в нанометрах и электронвольтах соответственно. В нашей работе эта формула была использована для расчета диаметров наночастиц $\mathrm{CdS}$ в синтезированных золях. Полученные значения $D$ частиц $\mathrm{CdS}$ в золях 1 и 2 после временной выдержки в течение $24 \mathrm{~h}$ при комнатной температуре составили 3.8 и $2.8 \mathrm{~nm}$ соответственно. Эти данные показывают, что в золях, стабилизированных низкомолекулярным ПВП, сформировались $\mathrm{CdS}$-частицы несколько меньшего размера.
Это явление может объясняться значительным различием в диффузионной подвижности полимерных молекул с различным молекулярным весом. Диффузионная подвижность молекул стабилизатора является важным фактором, так как скорость химической реакции в растворе между ионами кадмия и сульфид анионами является высокой. В присутствии относительно меньших по размеру молекул низкомолекулярного ПВП образование центров кристаллизации $\mathrm{CdS}$ протекает быстрее вследствие большей подвижности ПВП молекул и более низкой вязкости золя. Поэтому на стадии нуклеации в присутствии низкомолекулярного ПВП в золе формируется большое количество зародышей $\mathrm{CdS}$.

На следующей стадии роста частиц $\mathrm{CdS}$ концентрация оставшихся ионов кадмия и серы, а также мономеров $\mathrm{CdS}$ больше в золе, содержащем высокомолекулярный ПВП, что приводит к формированию в этом золе несколько более крупных $\mathrm{CdS}$-частиц.

В видимой части спектра потери света в полученных золях определяются его рассеянием. Наблюдаемые относительно высокие потери света в полученных золях в видимой части спектра не могут объясняться рассеянием света на сформировавшихся $\mathrm{CdS} / \mathrm{ZnS}$-частицах, так как их концентрация невелика и их размер составляет всего несколько нанометров. Золь 2, стабилизированный низкомолекулярным ПВП, демонстрирует более высокие потери света в видимом спектрально диапазоне, что может быть связано с формированием в нем относительно крупных агрегатов частиц.

Сопоставление серий кривых 1-3 (золь 1) и кривых 4-6 (золь 2) (рис. 1) показало, что увеличение продолжительности временной выдержки приводит к некоторому уменьшению прозрачности обоих золей в видимой части спектра. Характер наблюдаемых изменений в УФ спектральном диапазоне показал отсутствие заметного сдвига поглощения в длинноволновую часть спектра, что свидетельствовало бы о росте частиц при временной выдержке обоих золей. Поэтому наблюдаемое постепенное уменьшение прозрачности золей в видимой части спектра может быть приписано медленно протекающему процессу агрегации частиц.

Квантовые точки $\mathrm{CdS}$ характеризуются высокими нелинейно-оптическими свойствами, однако их характеристики сильно зависят от метода синтеза наночастиц, их концентрации, типа стабилизатора и структуры материала [6-8,15,16,24,30-32]. Результаты исследований синтезированных золей $\mathrm{CdS} / \mathrm{ZnS}$ в поле лазерного излучения в оптической схеме по методу $z$-сканирования приведены на рис. 2 и 3 для разных интенсивностей и разных составов стабилизатора. Как следует из спектров поглощения (рис. 1), излучение приходило в область прозрачности золей, поэтому нелинейное снижение пропускания при увеличении интенсивности в режиме открытой апертуры (рис. 2 и 3,a) обусловлено двухфотонным поглощением и нелинейным рассеянием. В режиме закрытой апертуры обнаружено проявление нелинейных рефрактивных свойств золей, действующих 


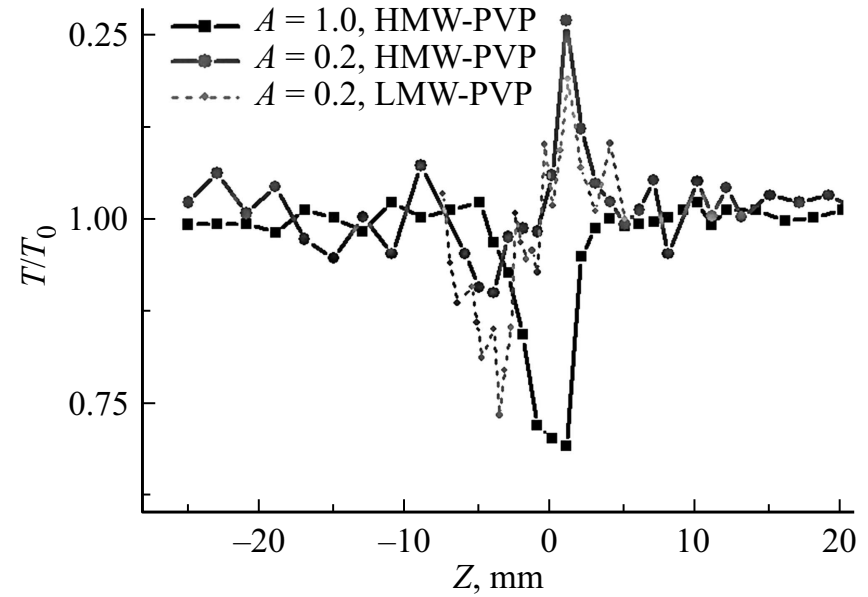

Рис. 2. Результаты $z$-сканирования золей при максимальной интенсивности излучения в фокусе $I_{F}=0.4 \mathrm{GW} / \mathrm{cm}^{2}:$, „HMW$\mathrm{PVP}^{\star}$ обозначены KT CdS/ZnS, стабилизированные высокомолекулярным ПВП, „LMW-PVP“ обозначены KT CdS/ZnS, стабилизированные низкомолекулярным ПВП. Значение $A=1$ соответствовало режиму открытой апертуры, $A=0.2-$ закрытой. $T_{0}-$ начальное линейное пропускание образца на длине волны $532 \mathrm{~nm}$.

как положительная линза (рис. 2 и 3,b). Рассчитанный по методу, предложенному авторами [24], показатель рефракции составил $|\gamma| \approx 4 \cdot 10^{-14} \mathrm{~cm}^{2} / \mathrm{W}(\gamma-$ нелинейный коэффициент рефракции связанных носителей, связан с нелинейным показателем преломления через соотношение $n_{2}=c n_{0} \gamma / 40 \pi$, здесь $n_{0}$ - линейный показатель преломления, $c$ - скорость света); коэффициент двухфотонного поглощения $\beta$ с вычетом влияния нелинейного рассеяния составил $4 \mathrm{~cm} / \mathrm{GW}$. Коэффициенты рассчитывались в приближении нелинейности третьего порядка для максимальной интенсивности в фокусе $I_{F}=0.4 \mathrm{GW} / \mathrm{cm}^{2}$, более детальный анализ нелинейных коэффициентов для золей на основе высокомолекулярного ПВП проведён нами ранее в [8]. Качественное сравнение нелинейно-оптических характеристик КТ $\mathrm{CdS} / \mathrm{ZnS}$, стабилизированных низко и высокомолекулярным ПВП показало, что преобладание содержания в золе высокомолекулярного ПВП приводит к увеличению нелинейных рефрактивных свойств (рис. 2,b), а низкомолекулярного - к увеличению рассеяния и двухфотонного поглощения (рис. 2,a). Однако величины рассчитанных нелинейных коэффициентов практически одинаковы для используемых концентраций КТ, их вариации находились в пределах погрешности эксперимента $\left(\varepsilon_{\text {отн }}=10 \%\right)$, основной вклад в которую вносила пространственная нестабильность лазерного пучка. Важно заметить, что золи $\mathrm{CdS} / \mathrm{ZnS}$ с низкомолекулярным ПВП малостабильны, в течение $10 \mathrm{~min}$ наночастицы выпадали в осадок с полной потерей нелинейных свойств. Установлен факт отсутствия нелинейного отклика самого ПВП при воздействии исходного лазерного излучения. Таким образом, в исследуемом диапазоне концентраций КТ молекулярный вес ПВП показал незначительное влияние на нелинейно-оптические свойства КТ.

Электронно-микроскопические снимки $\mathrm{CdS} / \mathrm{ZnS}-$ ПВП покрытий показаны на рис. 4. Рис. 4, $a, b$ демонстрируют различия в морфологии покрытий на уровне нескольких микрон. Покрытие, содержащее высокомолекулярный ПВП, является однородным и полностью покрывает поверхность подложки (рис. 4,a). На поверхности покрытия, содержащего низкомолекулярный ПВП, наблюдаются многочисленные трещины (рис. 4, $b$ ).

На электронно-микроскопических снимках, показанных на рис. 4, $c, d$, e видны частицы, имеющие размер около $100 \mathrm{~nm}$. Размер этих частиц значительно больше, чем размер частиц $\mathrm{CdS} / \mathrm{ZnS}$ в золях. Также можно отметить, что размер этих частиц примерно одинаков в обоих покрытиях.
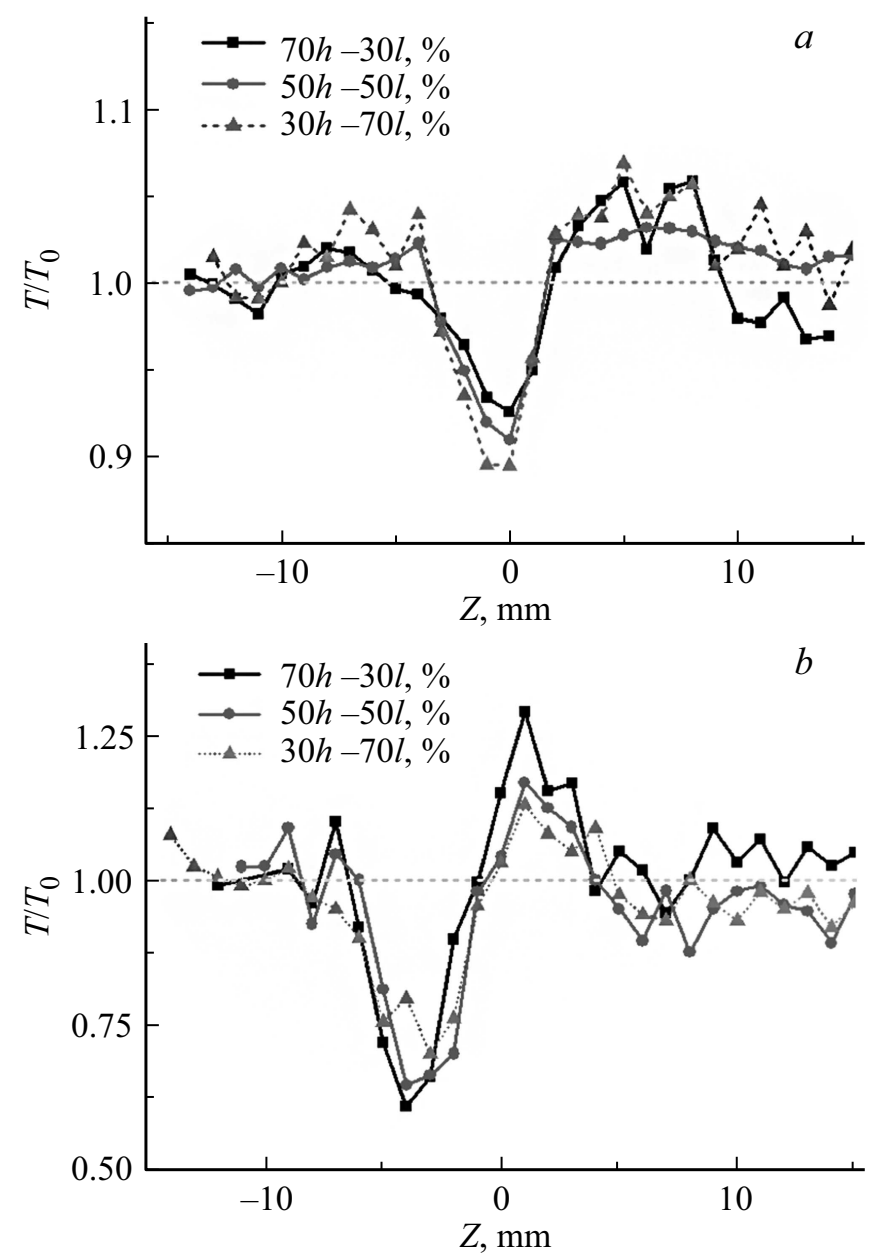

Рис. 3. Результаты $z$-сканирования золей при максимальной интенсивности излучения в фокусе $I_{F}=0.2 \mathrm{GW} / \mathrm{cm}^{2}: a-$ в режиме открытой апертуры, $b-$ в режиме закрытой. Составы стабилизаторов для КТ обозначены в соответствии с процентным содержанием низко- или высокомолекулярного ПВП, например золь „70h-301“ состоит из 70\% высокомолекулярного ПВП и $30 \%$ низкомолекулярного. 
$a$

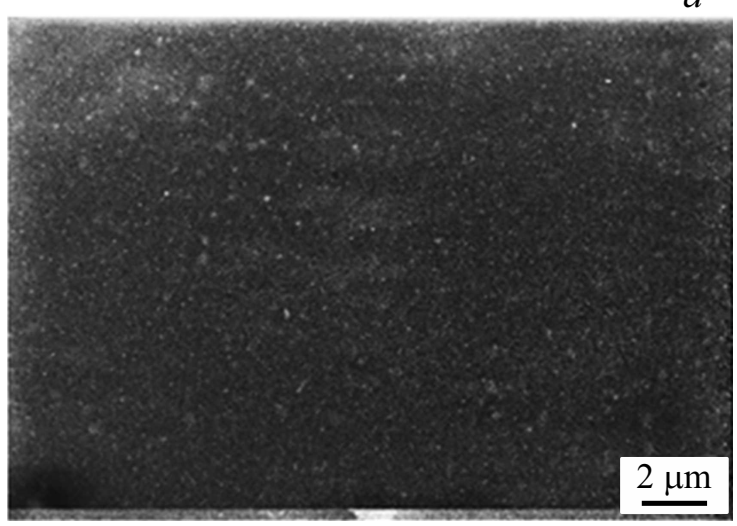

$c$

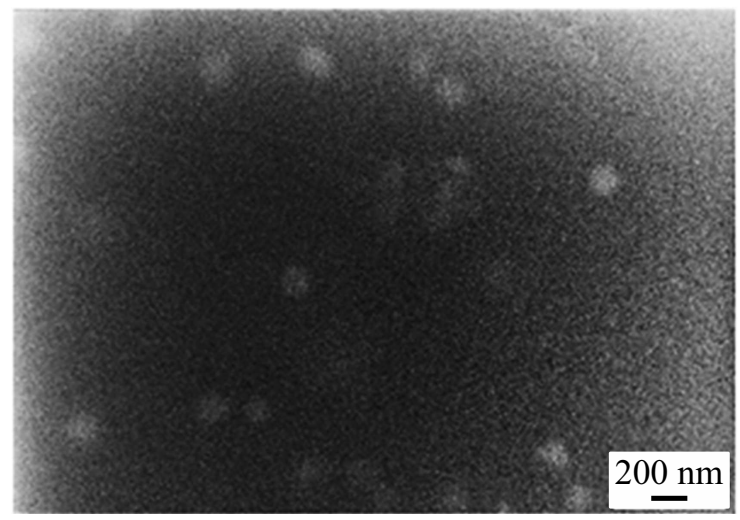

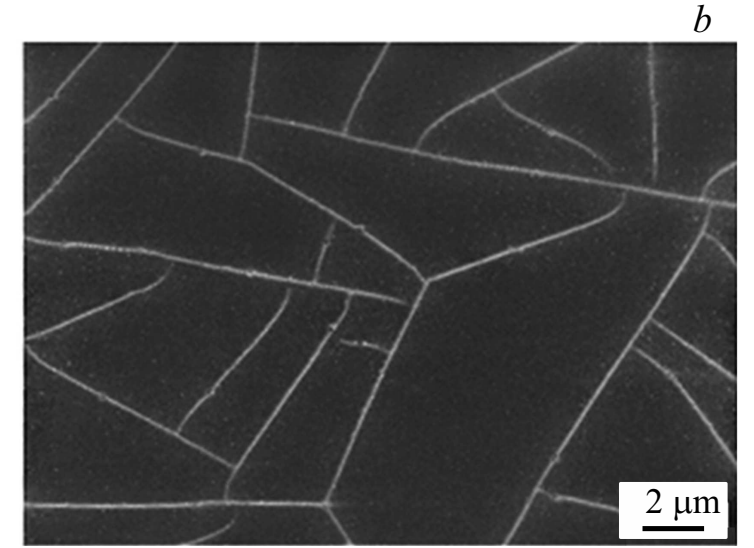

$d$

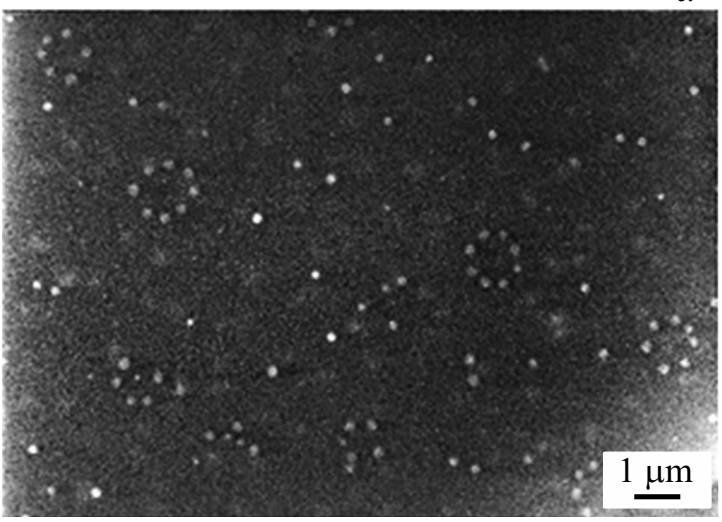

e

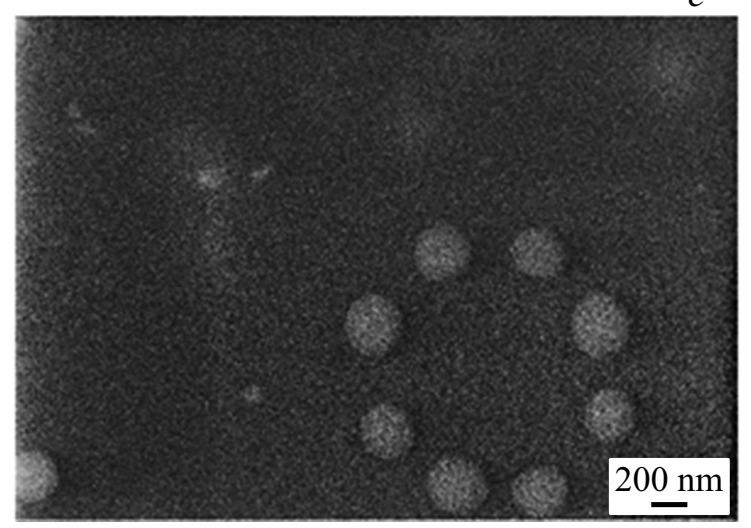

Рис. 4. Электронно-микроскопические снимки поверхности композиционных покрытий, изготовленных из золей 1 (a, $c)$ и $2(b, d, e)$.

Можно предположить, что эти частицы состоят из полупроводниковых наночастиц и молекул ПВП. Объемная доля ПВП в золях составляла только $1.4 \%$. Поэтому после сушки покрытий и удаления растворителей объемная усадка материала составила $\sim 98 \%$ и объемное содержание наночастиц возросло почти на два порядка. Столь значительное увеличение объемной концентрации наночастиц может приводить к формированию их относительно крупных агрегатов.

Частицы не образуют каких-либо агрегатов и случайным образом распределены между длинными цепями молекул высокомолекулярного ПВП в покрытии, пока- занном на рис. $4, b$. В отличие от этого в структуре композиционного покрытия на основе низкомолекулярного ПВП наблюдается формирование упорядоченных круговых ансамблей частиц (рис. $4, d, e)$. Эти ансамбли имеют форму „ожерелья“ и состоят из 7-8 отдельных частиц, разделенных друг от друга. Внутри ансамбля расстояния между соседними частицами примерно одинаковы.

Важная роль лигандов в процессах самоорганизации KT CdSe была продемонстрирована ранее в [33]. Различие в структуре полученных нами покрытий связано с большой разницей в размере молекул полимерного стабилизатора. Молекулы высокомолекулярного ПВП 
более эффективно препятствуют агрегации наночастиц. В процессе сушки покрытий, при одинаковом содержании остаточного растворителя, пленкообразующая композиция, содержащая высокомолекулярный ПВП, является существенно более вязкой, чем композиция на основе низкомолекулярного ПВП. Это определяет более низкую скорость пространственного перераспределения частиц и формирования структуры. Однако детальный механизм формирования круговых ансамблей частиц неясен и нуждается в дополнительном исследовании.

\section{Заключение}

Молекулярный вес полимерного стабилизатора поливинилпиролидона оказывает существенное влияние на структуру, спектральные и нелинейно-оптические свойства $\mathrm{CdS} / \mathrm{ZnS}$-золей и композиционных покрытий, полученных жидкостным методом. При коллоиднохимическом синтезе в золях, стабилизированных более низкомолекулярным поливинилпирролидоном, образуются более мелкие частицы CdS/ZnS. Использование высокомолекулярного поливинилпирролидона в качестве стабилизатора обеспечивает формирование в золе несколько более крупных наночастиц и значительно более эффективно препятствует процессам их агрегации и седиментации.

Даже разбавленные золи $\mathrm{CdS} / \mathrm{ZnS}$, стабилизированные высокомолекулярным поливинилпирролидоном, демонстрируют относительно высокие нелинейно-оптические свойства. Применение этих золей в качестве пленкообразующих композиций обеспечивает получение прозрачных однородных покрытий, которые могут быть перспективны в нелинейной оптике.

В покрытиях, полученных из золей $\mathrm{CdS} / \mathrm{ZnS}$, стабилизированных низкомолекулярным поливинилпирролидоном, наблюдается формирование ансамблей частиц, имеющих правильную круговую форму „ожерелья“.

Настоящая работа выполнена при частичной финансовой поддержки Министерства науки и образования Российской Федерации (проект RFMEFI58715X0012).

\section{Список литературы}

[1] Dabbousi B.O., Rodriguez-Viejo J., Mikulec F.V., Heine J.R., Mattoussi H., Ober R., Jensen K.F., Bawendi M.G. // J. Phys. Chem. B. 1997. V. 101. P. 9463.

[2] Mansur H.S., Mansur A.A.P., González J.C. // Polymer. 2011. V. 52. N 4. P. 1045.

[3] Kim Jae Ik, Kim Jongmin, Lee Junhee, Jung Dae-Ryong, Kim Hoechang, Choi Hongsik, Lee Sungjun, Byun Sujin, Kang Suji, Park Byungwoo // Nanoscale Res. Lett. 2012. $7: 482$.

[4] Thambiadurai M., Murugan N., Muthukumarasamy N., Agilan S., Vasantha S., Balasundaraprabhu R. // J. Mater. Sci. Technol. 2010. V. 26. N 3. P. 193.

[5] Sharma P., Rana D.S., Umar Ahmad, Kumar R., Chauhan M.S., Chauhan S. // Ceram. Int. 2016. V. 42. N 6. P. 6601.
[6] Venkatram N., Sai Santosh Kumar R., Narayana Rao D. // J. Appl. Phys. 2006. V. 100. P. 074309.

[7] Venkatram N., Narayana Rao D., Akundi M.A. // Optics Express. 2005. V. 13. N 3. P. 867.

[8] Кулагина А.С., Евстропьев С.К., Розанов Н.Н., Власов В.В. // ФТП. 2018. Т. 52. В. 8. С. 865.

[9] Багров И.В., Данилов В.В., Евстропьев С.К., Киселев В.М., Кисляков И.М., Панфутова А.С., Хребтов А.И. // Письма в ЖТФ. 2015. Т. 41. В. 2. С. 25.

[10] Евстропьев К.С., Гатчин Ю.А., Евстропьев С.К., Дукельский К.В., Кисляков И.М., Пегасова Н.А., Багров И.В. // Опт. и спектр. 2016. Т. 120. № 3. C. 434. Evstropév K.S., Gatchin Yu.A., Evstropév S.K., Dukelsśkii K.V., Kislyakov I.M., Pegasova N.A., Bagrov I.V. // Opt. Spectrosc. 2016. V. 120. N 3. P. 415.

[11] Bagrov I.V., Belousova I.M., Evstropiev S.K., Kislyakov I.M. // Polymers for Adv. Technol. 2015 V. 26. N 9. P. 1097.

[12] Pattabi M., Amma B.S., Manzoor K. // Mater. Res. Bull. 2007. V. 42. P. 828.

[13] Ghosh G., Naskar M.K., Patra A., Chatterjee M. // Opt. Mater. 2006. V. 28. N 8-9. P. 1047.

[14] Artemyev M.V., Gaponenko S.V., Germanenko I.N., Kapitonov A.M. // Chem. Phys. Lett. 1995. V. 243. P. 450.

[15] Jing Chengbin, Xu Xinguang, Zhang Xiaoliang Liu, Zhibo, Chu Junhao. // J. Phys. D: Appl. Phys. 2009. V. 42. N 7. P. 047402.

[16] Saravanan L., Diwakar S., Mohankumar R., Pandurangan A., Jayavel R. // Nanomater. Nanotechnol. 2011. V. 1. N 2. P. 42. www.intechweb.org.

[17] Chaudhuri T.K., Patel M.G. // Proceedings of the International Conference on Physics of Emerging Functional Materials (PEFM-2010). AIP Conference Proceedings. 2010. V. 1313(1) P. 275.

[18] Sato K., Kojima S., Hattori S., Chiba T., Ueda-Sarson K., Torimoto T., Tachibana Y., Kuwabata S. // Nanotechnology. 2007. V. 18. 465702.

[19] Niu Jinhong, Xu Weiwei, Shen Huaibin, Li Sen, Wang Hongzhe, Li Ling Song // Bull. Korean Chem. Soc. 2012. V. 33. N 2. P. 393.

[20] Liu Jinsong, Zhao Chuanbao, Li Ziquan, Chen Jiankang, Zhou Hengzhi, Gu Shanqun, Zeng Youhong, Li Yongchan, Huang Youngbing // J. Alloys and Compounds. 2011. V. 509. P. 9428.

[21] Kim Mee Rahn, Kang Young-Mee, Jang Du-Jeon // J. Phys. Chem. C. 2011. V. 111. P. 18507.

[22] Vempati S., Ertas Y., Uyar T. // J. Phys Chem C. 2013. V. 117. P. 21609.

[23] Evstropiev S.K., Kislyakov I.M., Bagrov I.V., Belousova I.M. // Polymers for Adv. Technol. 2016. V. 27. P. 314. doi 10.1002/pat.3642

[24] Said A.A., Sheik-Bahae M., Hagan D.J., Wei T.H., Wang J., Young J., Stryland V.E.W. // J. Opt. Soc. Am. B. 1992. V. 9. P. 405.

[25] Williams R. // J. Phys. Chem. Solids. 1962. V. 23. P. 1057.

[26] Sooklal K., Cullumn B.S., Angel S.M., Murphy C.J. // J. Phys. Chem. 1996. V. 100. P. 4551.

[27] Tauc J. // Mater. Res. Bull. 1968. V. 3. N 1. P. 37.

[28] Kozhevnikova N.S., Vorokh A.S., Uritskaya A.A. // Russian Chem. Rev. 2015. V. 84. N 3. P. 225.

[29] Эфрос Ал.Л., Эфброс А.Л. // ФТП. 1982. Т. 16. № 7. С. 1209. 
[30] Jing Chengbin, $X u$ Xinguang, Zhang Xiaoliang, Chu Junhao // J. Phys. D: Appl. Phys. 2009. V. 42. 075402 (6pp).

[31] Li H.P., Kam C.H., Lam Y.L., Ji W. // Opt. Comm. 2001. V. 190. N 1-6. P. 351.

[32] Du H., Xu G.Q., Chin W.S. // Chem. Mater. 2002. V. 14. P. 4473.

[33] Ushakova E.V., Kormilina T.K., Burkova M.A., Cherevkov S.A., Zakharov V.V., Turkov V.K., Fedorov A.V., Baranov A.V. // Opt. Spectrosc. 2017. V. 122. N 1. P. 25. 